\title{
Atomic Energy as well as Gravitational Energy is Another form of Electrical Energy
}

\author{
Manish Kumar \\ Department of Electrical Engineering, Indian Institute of Technology (Banaras Hindu University), Varanasi-221005, U.P., INDIA
}

Received: November 22, 2016 / Accepted: December 20, 2016 / Published: February 25, 2017.

\begin{abstract}
Atomic energy is the source of power which is another form of electrical energy. This electrical energy is basically the fundamental energy which is the source of all other energy known to world. We can say that from the prediction of genius Albert Einstein for the unification of forces it is the electrical force that is the root cause of all other forces. When a charge particle moves it produces magnetic force i.e. electrical energy is converted to magnetic energy and charge particle has only electrical energy only. It is the movement of electrical energy that leads to generation of magnetic energy according to the Maxwell's law. Since these forces are long range forces so when they follow the Maxwell's law leads to generation of electromagnetic radiation. The weak and strong nuclear forces discovered which is the cause for binding sub-atomic particles into the nucleus is nothing but the electrical energy being converted into sub-atomic particles due to the conversion of electrical energy into mass with the electrical charge of positive and negative character depending upon the orientation of electrical energy into clockwise or anticlockwise direction. The packing of electrical energy into mass is governed by the anti-law that leads the conversion of mass into energy in the atomic energy given by Einstein's famous relation $\mathrm{E}=\mathrm{mc}^{2}$.
\end{abstract}

\section{Introduction}

"Necessity is the mother of invention" is the famous proverb which originates from the inquisitive mind of human beings. From paleotheolic age to modern world the fight for survival is the necessity that lead to the consequential development of modern civilization. Although almost all theories of nature have been discovered which correctly explains the Universe which are in the limits of Physics. The most revolutionary theories came from those who has undeterred faith in GOD. Till now there was lot of debate on the existence of GOD as there was no way to prove the existence of GOD scientifically. The only proof of GOD was the undeterred faith of human beings which was being challenged by many atheist those who deny His complete existence and hence the debate leads to many war and bloodshed. Recently

Corresponding author: Manish Kumar, Department of Electrical Engineering, Indian Institute of Technology (Banaras Hindu University), Varanasi-221005, U.P., INDIA.
GOD's existence was proved scientifically by Kumar [1] using the mathematics as the diagnostic tool. GOD's physical existence and his interaction with the matter (living/non-living) is beyond the realm of physics as the boundary of physics is $\hbar / 2$. Another proof of GOD's communication with the living/non-living matter was given by Kumar et al [2]. In this paper Kumar et al has shown that any radiation falling on the rippled surface under the presence of magnetic field get converted into surface magnetoplasmons, so from this inference can be easily drawn how life is affected by universal phenomenon as every matter radiates its own energy in the form of radiation depending upon its characteristic and thus affect the life. Understanding the natural philosophy till now is being the consequence of having undeterred faith in GOD causing the emergence of pioneers and advancements made through the effort of various scientists and researchers, these fields as oneself came to light as a path of research in their own right.Different potential ideas in physics has utilized 
the mathematics as the diagnostic tool and various prescription came from sectors like Classical Physics, Modern Physics, Computational Physics, Theoretical Physics, Applied Physics, Meta Physics, Solar Physics, Bio Physics, Astro Physics etc. for creation, invention and discovery as the Physics is the study of science that deals with energy and matter and their interactive nature with each other.

Some more proofs regarding the GOD's existence and His interaction is described in following sections:

\section{Proof from Quantum Physics}

Kumar [1] in his paper shows from the uncertainty principle and using mass-energy equivalence that GOD does exist. From the simple mathematical proof which for easy reference is shown below

$$
\therefore E(\Delta x)^{2} / \Delta t=0
$$

This implies either of the three conditions which are as follows:

$\begin{array}{rlrl} & & E & =0 \\ \text { or } & \Delta x & =0 \\ \text { or } & & \end{array}$

$$
\Delta t=\infty
$$

If $E=0$ then there is no discussion, so we can rule out this possibility or this also implies that when there is nothing still there is GOD i.e. everything in the universe/multiverse is originated from "Zero" which also describes GOD's characteristics. If $\Delta x=0$, then it implies GOD's position does not change or it can be said that GOD is everywhere in the universe/multiverse so this is also true for GOD. Third possibility is $\Delta t=\infty$

i.e. time period is infinite, then also our hypothesis is justified. It is what GOD is, whose time period is infinite, rest all the quantities in the universe/multiverse are having finite time period.

Also the first condition that $\mathrm{E}=0$, means that the positive energy and the negative energy are in exact phase matching thus resulting in zero energy. Here the positive energy means the man soul and the negative energy means the female soul. So the conclusion is that universe was created with only two species male and female in any living matter. So the purpose of life is to understand this creation which are explained in below sections.

\section{Proof from Astro Physics}

Consider the atomic model an electron circulating the nucleus containing proton i.e. Hydrogen atom model. There are two attractive forces i.e. gravitational attraction because of masses of proton, electron and the coulomb attraction because of opposite charges of the two particles. These forces are balanced by the centrifugal force because of the revolution electron around the proton viz.

$$
m_{e} v^{2} / r=G m_{p} m_{e} / r^{2}+e^{2} /\left(4 \pi \varepsilon_{0} r^{2}\right)
$$

Where $m_{e}, m_{p}$ is the mass of electron, proton respectively, $e$ is charge of electron and proton, $G$ is the Universal Gravitational Constant, $r$ is radius of orbit in which electron revolves around the proton or nucleus and $v$ is the orbiting velocity of electron.

Also $I=-e / t, t=2 \pi r / v \Rightarrow v=-I 2 \pi r / e$

$$
H=I / 2 r \Rightarrow I=H 2 r
$$

Substituting all this into force equation one obtains

$$
\begin{gathered}
H= \pm\left[\frac{G m_{p} e^{2}}{16 \pi^{2} r^{5}}+\frac{e^{4}}{64 \pi^{3} \varepsilon_{0} m_{e} r^{5}}\right]^{1 / 2} \\
r=\left[\frac{G m_{p} e^{2}}{16 \pi^{2} H^{2}}+\frac{e^{4}}{64 \pi^{3} \varepsilon_{0} m_{e} H^{2}}\right]^{1 / 5}
\end{gathered}
$$

Where $\mathrm{H}$ is the magnetic field intensity. The above two equations show that $\mathrm{H}$ is not only the consequence current but also the mass of the two particles play role. Now using similarity of above model for the universe replacing the mass of proton with the mass of GOD at the centre of Universe and mass of electron as mass of the Universe circulating around it having single positive and negative charge respectively one obtains 


$$
\begin{aligned}
& H= \pm\left[\frac{G M_{G O D} e^{2}}{16 \pi^{2} r^{5}}+\frac{e^{4}}{64 \pi^{3} \varepsilon_{0} M_{\text {UNVERSE }} r^{5}}\right]^{1 / 2} \\
& r=\left[\frac{G M_{G O D} e^{2}}{16 \pi^{2} H^{2}}+\frac{e^{4}}{64 \pi^{3} \varepsilon_{0} M_{\text {UNVERSE }} H^{2}}\right]^{1 / 5}
\end{aligned}
$$

Since Astrophysics has proved the existence of constant magnetic field in the Universe and has also measured to be about $10^{-13}$ Tesla. So by knowing the masses we can determine the distance of GOD from the Universe. Also a very interesting result can be derived from here just by considering the mass energy equivalence as given by the $E=m c^{2}$ $\Rightarrow \quad m=E / c^{2}$, where $m, c$ are mass of any object, velocity of light which according to Albert Einstein is absolute and is a constant in any frame of reference respectively and so $E$ is the corresponding energy associated with the object.

$$
\begin{aligned}
& H= \pm\left[\left(\frac{G e^{2}}{16 \pi^{2} r^{5} c^{2}}\right) E_{G O D}+\left(\frac{e^{4} c^{2}}{64 \pi^{3} \varepsilon_{0} r^{5}}\right) \frac{1}{E_{\text {UNIVERSE }}}\right]^{1 / 2} \\
& \left(\frac{H}{D}\right)^{2}=\left(\frac{G}{c^{2} r}\right) E_{G O D}+\left(c^{2} r F_{\text {Elect }}\right) \frac{1}{E_{\text {UNIVERSE }}}
\end{aligned}
$$

Where

$$
D=e / 4 \pi r^{2} \& F_{\text {Elect }}=e^{2} / 4 \pi \varepsilon_{0} r^{2}
$$

Gravitational energy is another form of Electrical energy which always exist between GOD and Universe/Multiverse as they are always opposite in nature as defined earlier as positive (male) and negative (female) energy also clear from the above derived equation and hence always attractive in nature but since it changes from positive to negative and hence results in gravitational waves i.e. gravity and anti-gravity which is also experimentally verified to exist in universe/multiverse. This leads to unification of forces i.e. the fundamental force is Electrical Force between GOD and universe/multiverse which causes electromagnetic as well as nuclear forces to bind the sub-atomic particles to all the masses in the universe/multiverse.

\section{Proof from Meta Physics}

Meta Physics is that branch of physics which deal with the spiritual part of life. Here we would like to quote few versus from Shri MadBhagwad Gita, a holy book of Hindu mythology which is supposed to give all the guidelines for humanity and is accepted across whole the globe viz.

Chapter 2: Contents of the Gītā Summarized

TEXT 22

vasamsi jirnani yatha vihaya

navani grhnati naro 'parani

tatha sarirani vihaya jirnany

anyani samyati navani dehi

\section{SYNONYMS}

vāsā $\dot{m}_{s i-g a r m e n t s ;}$ jīrṇanni-old and worn out; yath $\bar{a}$-as it is; vihāya-giving up; navāni-new garments; g!̣nhāti-does accept; naraḥ-a man;aparāni-other; tath $\bar{a}-$ in the same way; śarìrāni-bodies; vihāya-giving up;jīrṇāni-old and useless; anyāni-different; samyāti-verily accepts; navāni-new sets; dehī-the embodied.

\section{TRANSLATION}

As a person puts on new garments, giving up old ones, similarly, the soul accepts new material bodies, giving up the old and useless ones.

\section{PURPORT}

Change of body by the atomic individual soul is an accepted fact. Even some of the modern scientists who do not believe in the existence of the soul, but at the same time cannot explain the source of energy from the heart, have to accept continuous changes of body which appear from childhood to boyhood and from boyhood to youth and again from youth to old age. From old age, the change is transferred to another body. This has already been explained in the previous verse.

Transference of the atomic individual soul to another body is made possible by the grace of the Super soul.The Super soul fulfills the desire of the atomic soul as one friend fulfills the desire of another. 
This is clear indication of interaction of matter with energy as the soul is basically the GOD's energy which drives all the life in Universe. Now here comes the mathematics as a diagnostic tool. It is very clear from the Maxwell's equation is that all radiation is electromagnetic in nature which are governed by those equation which is a pure result of Mathematics and the prescription i.e. Physics derived from here results in the development of Modern world. Now we would like to quote another sloke of Gita then we shall elaborate its consequence in work:

The verse is-

कर्मण्येवाधिकारस्तेमाफलेषुकदाचन।

माकर्मफलहेतुर्भूर्मातेसङ्गोऽस्त्वकर्मणि॥२-४७

In Roman scripts-

Karmanye vadhikaraste Ma Phaleshu Kadachana,

Ma Karmaphalaheturbhurma Te Sangostvakarmani

The meaning of the verse is -

You have the right to work only but never to its fruits.

Let not the fruits of action be your motive, nor let your attachment be to inaction.

Now let us allow to elaborate this in connection with the properties of Electrical and Magnetic force. As is very clear that Electrical force can accelerate or decelerate the charge particles but the effect of Magnetic force is to only change the direction of charge particle, also note that it is the Electrical field that is the cause of Magnetic field. So we relate the Karma as the Electric force and the luck as the magnetic force which is the result of karma or Electrical force. So we have only right to work and hence not in the result because result or luck is the consequence of it. Now what connection it has with the previous verse. It is an established fact that person is born with different fates i.e. the soul changes body as we change clothes so the karma done in previous life is converted into luck in its new life. Now a very important question arises that how the previous karma is affecting the current life? How the soul is affected by the karma and how this information of one life is carried to another life via soul? To explain this we need to understand some mathematics which is simple to understand as only mathematics provide a scientifically correct information which cannot be challenged. Assume that every person is designated by some complex number i.e. one's work can be expressed into complex number hence the person will have maximum magnitude and phase if that complex number is expressed in polar form. So the magnitude represent the maximum work one can carry out then the phase of that number is basically the desires of a person or we can righteously say this "as a feeling of that person". So one basically has control over one's feelings only as the work of each person is fixed but both the work or the amplitude and the phase are functions of time and space. This is the reason every person is affected by the whole universal phenomenon consciously or unconsciously. Since any action or work performed by any person is initiated by one's feeling or thoughts. If internal force is not driving that feeling or thought no action will be performed this is universal fact accepted by theist or atheist even after an intense provocation. Since there are only two needs that drive the life firstly the biological and secondly psychological. Biological need is to fulfil the requirements of body and the psychological need is food for thought/feelings/desires whatever one call. So every person is driven by these needs and one's action is purely a reflection of these needs. Even if person is educated/uneducated has inherent quality of fulfilling ones basic needs as soon as these basic needs are fulfilled other need drives one for fulfilling other need and this is called as consciousness of person. This consciousnesscannot be of any non-living thing. The limit of this consciousness is Salvation or Moksha which according to Hindu mythology is the reason of creation of universe by the GOD. This knowledge has been imparted in Gita and the means to achieve has also been described very clearly which is accepted globally. So we are not going in philosophical description of achieving this goal rather we will focus 
on more methodological and scientific approach for it which put a final stamp on End of Physics.

With this goal again we comeback on person being represented by a complex function in which one amplitude as well as phase is function of space and time, since we are assumed to be a journey hence frequency and the propagation constant is also evolving with the time and space assumed to be complex i.e.

$$
\omega=\omega_{r}+i \omega_{i}, k=k_{r}+i k_{i}
$$

From the knowledge of wave theory that phase velocity $V_{P}=\omega / k$ and the group velocity $V_{G}=\partial \omega / \partial k$ are related as $V_{P} V_{G}=c^{2}$, where $c$ is the speed of light and till now is accepted as universal constant in any frame of reference and is independent of velocity of source. So substituting the value of $\omega$ and $k$ in phase velocity and group velocity and simplify one get

$$
\begin{aligned}
& \frac{\left(\omega_{r} k_{r}+\omega_{i} k_{i}\right)}{\left(k_{r}^{2}+k_{i}^{2}\right)} \frac{\left(\frac{\partial k_{r}}{\partial \omega_{r}}+\frac{\partial k_{i}}{\partial \omega_{i}}\right)}{\left(\left(\frac{\partial k_{r}}{\partial \omega_{r}}\right)^{2}+\left(\frac{\partial k_{i}}{\partial \omega_{i}}\right)^{2}\right)} \\
& -\frac{\left(\omega_{i} k_{r}-\omega_{r} k_{i}\right)}{\left(k_{r}^{2}+k_{i}^{2}\right)} \frac{\left(\frac{\partial k_{r}}{\partial \omega_{i}}-\frac{\partial k_{i}}{\partial \omega_{r}}\right)}{\left(\left(\frac{\partial k_{r}}{\partial \omega_{i}}\right)^{2}+\left(\frac{\partial k_{i}}{\partial \omega_{i}}\right)^{2}\right)} \\
& +i\left(\begin{array}{c}
\frac{\left(\omega_{i} k_{r}-\omega_{r} k_{i}\right)}{\left(k_{r}^{2}+k_{i}^{2}\right)} \frac{\left(\frac{\partial k_{r}}{\partial \omega_{r}}+\frac{\partial k_{i}}{\partial \omega_{i}}\right)^{2}}{\left(\left(\frac{\partial k_{r}}{\partial \omega_{r}}\right)^{2}+\left(\frac{\partial k_{i}}{\partial \omega_{i}}\right)^{2}\right)} \\
\left.+\frac{\left(\omega_{r} k_{r}+\omega_{i} k_{i}\right)}{\left(k_{r}^{2}+k_{i}^{2}\right)} \frac{\left(\frac{\partial k_{r}}{\partial \omega_{i}}-\frac{\partial k_{i}}{\partial \omega_{r}}\right)^{2}}{\left(\left(\frac{\partial k_{r}}{\partial \omega_{i}}\right)^{2}+\left(\frac{\partial k_{i}}{\partial \omega_{i}}\right)^{2}\right)}\right)
\end{array}\right)=c^{2}
\end{aligned}
$$

From here we come to conclusion that indeed that speed of light is not real but a complex quantity which instantly reveals that indeed understanding GOD is beyond the realm of Physics.

\section{Proof from Bio-Physics}

Bio-Physics just like any other branch of physics plays important role in the concept of energy. We can simply conclude that everything is physics, and it plays important role in our life. Taking simple illustration of a child born. Till now there has been no evidence of whether the child born will be male or female or the third gender in the pre-initialstage (zygote formation stage).So one cannot determine the gender of child in initial stage. But with the new concept we can easily know the sex of the child. With the concept of realization of creation of universe, the positive and negative energy are two part of universe. The positive representing the male soul and the negative energy represents the female soul. Since the gender of new born is decided by the chromosome pair XX and XY, XX being from female egg and XY from male sperm. So when sperm and egg fuse, there is probability that new born will either get $\mathrm{XX}$ or $\mathrm{XY}$ from fusion, this lay the formation of zygote or the new baby. Now this is the moment when actually a soul interacts with matter i.e. body. Triggering means energy matter interaction. Energy means soul which can either be positive or negative. Ultimate aim of life is salvation not mating. And this is obtained by triggering. And this has even been incorporated in even our Vedas. The salvation only is obtained when positive and exact negative meet it becomes zero i.e. GOD. So in conclusion this is Life an answer to our great founder of Quantum Physics Erwin Schrodinger who said"What is Life?" [3].

\section{Conclusion}

It is now very clear from all the mathematical diagnosis and the physics being derived from the mathematical solutions from various sectors like Classical Physics, Modern Physics, Computational Physics, TheoreticalPhysics, Applied Physics, Meta Physics, Solar Physics, Bio Physics, Astro Physics etc. 


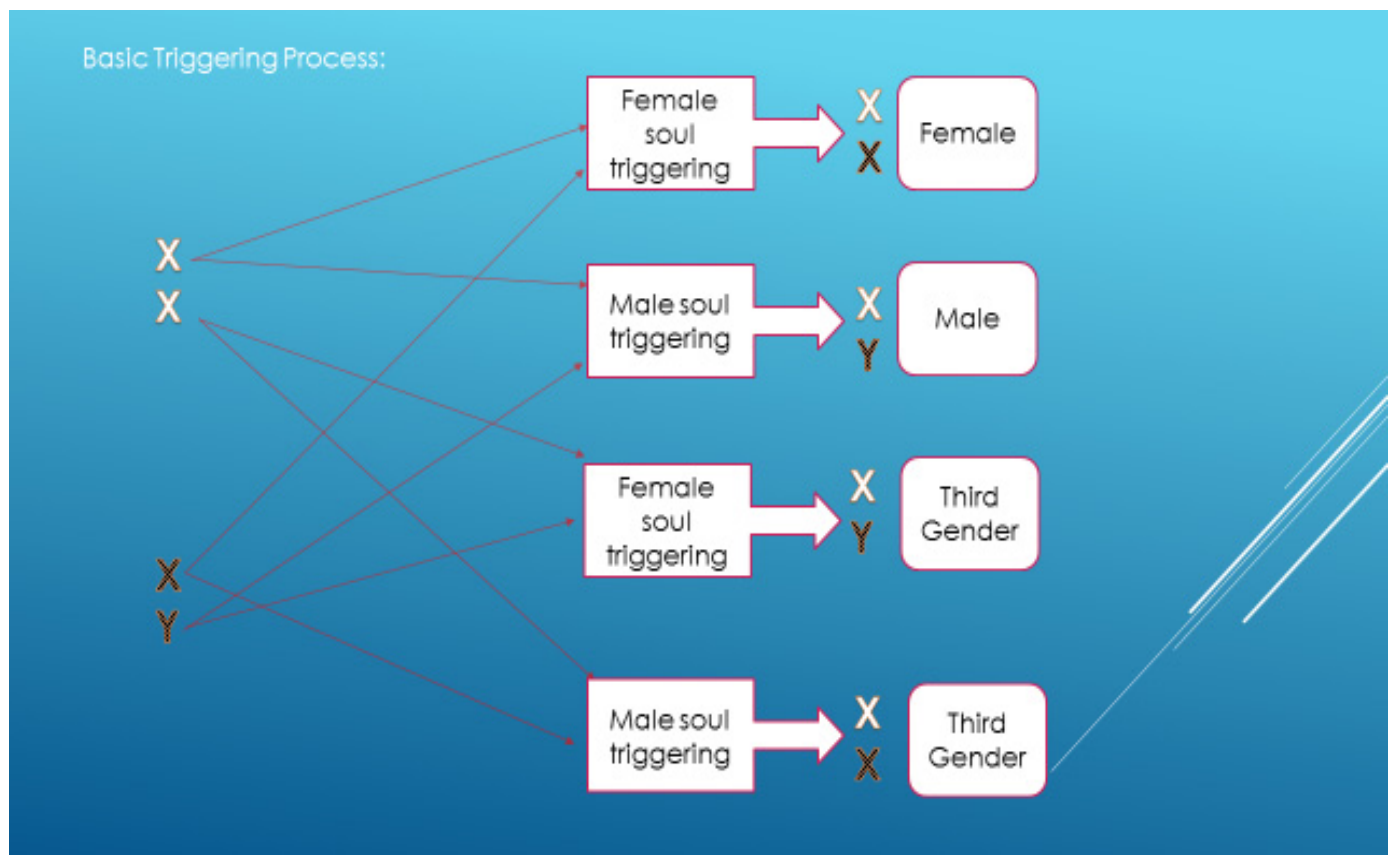

for creation, invention and discovery, are leading to similar conclusions what various holy books and scriptures available throughout the world conclude that is GOD does exist and is one. GOD can only be realized by humans and so are His creations, with their ultimate goal being is salvation.Atomic as well as gravitational energy is another form of electrical energy. This electrical energy is basically the fundamental energy which is the source of all other energy known to world. We can say that from the prediction of genius Albert Einstein for the unification of forces it is the electrical force that is the root cause of all other forces. When a charge particle moves it produces magnetic force i.e. electrical energy is converted to magnetic energy and charge particle has only electrical energy only. It is the movement of electrical energy that leads to generation of magnetic energy according to the Maxwell's law. Since these forces are long range forces so when they follow the Maxwell's law leads to generation of electromagnetic radiation. The weak and strong nuclear forces discovered which is the cause for binding sub-atomic particles into the nucleus is nothing but the electrical energy being converted into sub-atomic particles due to the conversion of electrical energy into mass with the electrical charge of positive and negative character depending upon the orientation of electrical energy into clockwise or anticlockwise direction. The packing of electrical energy into mass is governed by the anti-law that leads the conversion of mass into energy in the atomic energy given by Einstein's famous relation $\mathrm{E}=\mathrm{mc}^{2}$.

\section{References}

[1] "GOD doesn't play dice", Manish Kumar, International Journal of Scientific \& Engineering Research,Volume 7, Issue 1, January 2016 Edition.

[2] "Linear mode conversion of $\mathrm{THz}$ radiation into $\mathrm{THz}$ surface magnetoplasmons on a rippled surface of magnetized n-InSb", Pawan Kumar, Manish Kumar and V. K. Tripathi, 1408 Vol. 41, No. 7 / April 12016 / Optics Letters.

[3] "What is Life?" Erwin Schrodinger, ISBN 978-1-107-60466-7, Cambridge University Press, The Edinburgh Building, Cambridge CB2 8RU, UK 